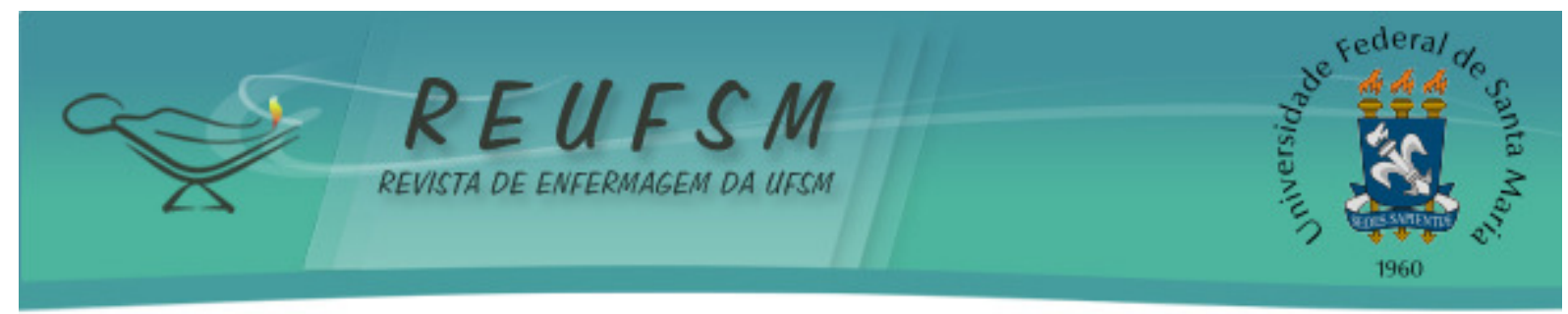

ARTIGO ORIGINAL

\title{
CARACTERIZAÇÃO DAS QUEDAS DE PACIENTES EM HOSPITAL ESPECIALIZADO EM CARDIOLOGIA
}

\section{CHARACTERIZATION OF PATIENT'S FALLS IN A CARDIOLOGY HOSPITAL CARACTERIZACIÓN DE LAS CAÍDAS DE PACIENTES EN HOSPITAL ESPECIALIZADO EN CARDIOLOGÍA}

Doi: 10.5902/2179769213554

\author{
Silmara Meneguin ${ }^{1}$ \\ Jairo Aparecido Ayres ${ }^{2}$ \\ Giovanna Hass Bueno ${ }^{3}$
}

RESUMO: Objetivo: Caracterizar as quedas de pacientes, segundo registro de eventos adversos, em unidades de internação de hospital especializado em cardiologia, do município de São Paulo. Método: estudo retrospectivo em que foram analisadas descritivamente 67 notificações de eventos adversos ocorridas no primeiro semestre de 2007. Resultados: registraram-se 2,04 quedas/mil paciente-dia, a maioria do sexo masculino $(59,7 \%)$, idade média 64,04 anos, ocorridas nos primeiros 30 dias da internação $(58,2 \%)$ e no período noturno $(55,2 \%)$. Quanto ao local, quarto e banheiro obtiveram maior destaque, $73,1 \%$ e $22,4 \%$, respectivamente. A queda da própria altura contribuiu com $64,2 \%$ dos casos, de poltronas $18,0 \%$ e do leito $10,4 \%$. Conclusões: os resultados desse estudo denotam a necessidade de maior cuidado com os idosos internados, durante o período noturno, no quarto e especialmente nos primeiros trinta dias de internação.

Descritores: Acidentes por quedas; Cuidados de enfermagem; Indicadores de qualidade em assistência à saúde; Cardiologia.

ABSTRACT: Objective: To characterize patient falls, according to records of adverse events, in wards of a cardiology hospital in the city of São Paulo. Method: retrospective study in which a descriptive analysis was carried out with 67 reports of adverse events occurred in the first semester of 2007. Results: 2.04 falls/ 1.000 patients-day were reported. Most patients were male (59.7\%), at mean age of 64.04 years old. The falls occurred in the first 30 days of hospitalization (58.2\%) at night (55.2\%). Concerning the places where the falls occurred, the bedroom and the bathroom showed the highest prevalence, $73.1 \%$ and $22.4 \%$, respectively. Falls from one's own height contributed with $64.2 \%$ of the cases, from armchairs $18.0 \%$, and from bed $10.4 \%$. Conclusions: the results of the present study point out the need for more care with elderly patients, at night, in the bedroom and especially during the first thirty days of stay.

Descriptors: Accidental falls; Nursing care; Nursing care; Quality indicators, health care; Cardiology.

RESUMEN: Objetivo: Caracterizar las caídas de pacientes, según registro de eventos adversos, en unidades de internación de hospital especializado en cardiología, del municipio de São Paulo. Método: estudio retrospectivo en que fueron analizadas descriptivamente 67 notificaciones de eventos adversos ocurridas en el primer semestre

\footnotetext{
${ }^{1}$ Enfermeira. Doutora em Ciências. Curso de Enfermagem - Faculdade de Medicina de Botucatu/UNESP. Botucatu, São Paulo, Brasil. E-mail:silmeneguin@fmb.unesp.br

${ }^{2}$ Enfermeiro. Doutor em Doenças Tropicais. Curso de Enfermagem - Faculdade de Medicina de Botucatu/UNESP. Botucatu, São Paulo, Brasil. E-mail:ayres@fmb.unesp.br

${ }^{3}$ Enfermeira. Egressa do Curso de Enfermagem - Faculdade de Medicina de Botucatu/UNESP. Botucatu, São Paulo, Brasil. E-mail:giovannahass@uol.com.br
} 


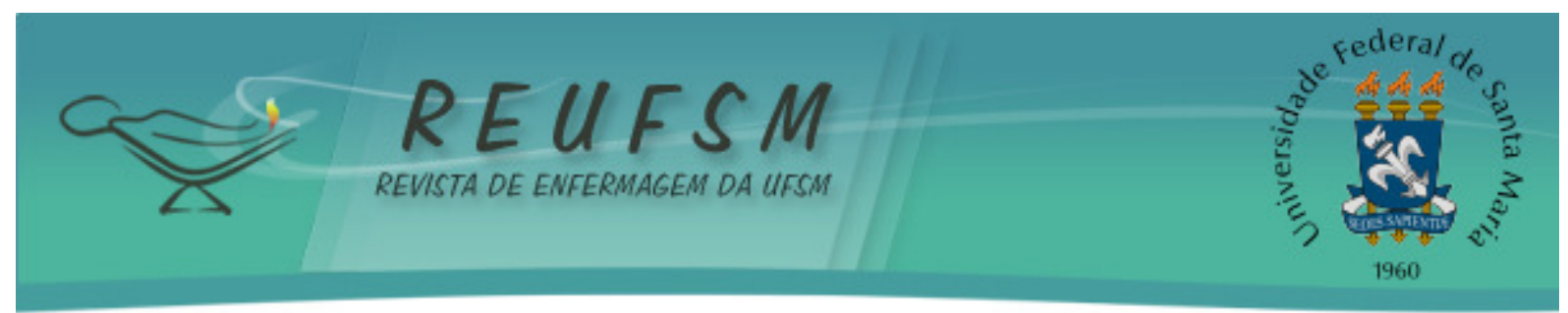

de 2007. Resultados: fueron registradas 2,04 caídas/mil pacientes-día, la mayoría do sexo masculino (59,7\%), edad promedia 64,04 años, ocurridas durante los primeros 30 días de hospitalización $(58,2 \%)$ y por la noche $(55,2 \%)$. Respecto al lugar, la habitación y el baño se destacaron con $73,1 \%$ y $22,4 \%$, respectivamente. La caída de la propia altura contribuyó con el $64,2 \%$ de los casos, de sillones $18,0 \%$ y de la cama 10,4\%. Conclusiones: los resultados de este estudio expresan la necesidad de extremar cuidados con los ancianos internados, durante el período nocturno en los cuartos, especialmente en los primeros treinta días de internación.

Descriptores: Accidentes por caídas; Atención de enfermería; Indicadores de calidad de la atención de salud; Cardiología.

\section{INTRODUÇÃO}

O crescimento da população idosa é fato mundial que merece atenção, pois sabese que a qualidade de vida pode ser comprometida pela perda de autonomia e independência. No Brasil, consideram-se as pessoas socialmente e cronologicamente idosas com idade igual ou superior a 60 anos. ${ }^{1}$ Por ser fenômeno dinâmico e progressivo, o envelhecimento causa alterações estruturais, fisiológicas, psicológicas e sociais ao indivíduo, tornando-o susceptível a processos patológicos e à ocorrência de quedas. ${ }^{2-3}$

Estudos têm mostrado que as quedas representam os eventos adversos mais prevalentes do âmbito hospitalar ${ }^{4-5}$ e proporcional à idade. ${ }^{6}$ Os índices oscilam entre 1,4 e 10,7 quedas para cada 1,000 pacientes-dia, dependendo da instituição hospitalar e do perfil dos pacientes. ${ }^{7}$

Doenças neurológicas e cardiovasculares estão muitas vezes associadas à ocorrência de quedas em virtude do processo fisiopatológico e tratamento inerentes a estas doenças. ${ }^{6}$ A queda em idosos afeta a qualidade de vida deste grupo populacional em decorrência de circunstâncias desencadeadas pela imobilidade e dependência. Além disso, também contribui para o aumento da mortalidade, principalmente por fraturas que necessitam de intervenções cirúrgicas.

A fratura de fêmur é uma importante causa de internação hospitalar pelo Sistema Único de Saúde (SUS) e ocorre em aproximadamente um quarto das fraturas pós-quedas, aumentando os custos sociais e econômicos. ${ }^{8}$ Nos Estados Unidos são gastos anualmente pelo sistema de saúde cerca de 10 bilhões de dólares para tratamento de fratura de quadril. ${ }^{9}$

Nesse contexto, a prevenção de queda requer o conhecimento dos fatores de risco que propiciam esse evento, pois são multifatoriais e complexos, podem resultar em transtornos como hospitalização, alto custo do tratamento e morte. ${ }^{10-11}$

Considerando que o processo de cuidar envolve a convergência das ações de enfermagem para o atendimento das necessidades do paciente, a implementação de medidas preventivas relacionadas às quedas está condicionada também à análise dos indicadores de qualidade assistencial de enfermagem, como a incidência de queda.

A utilização de indicadores traz como benefício não somente a possibilidade de acompanhamento da qualidade da assistência de enfermagem no âmbito institucional, mas também permite a comparação dos escores entre os hospitais. Além disso, fornece subsídios para avaliação hospitalar e tomada de decisão pelos gestores. ${ }^{12}$

Espera-se que a presente análise traga contribuições para implementação e intensificação de ações preventivas específicas e de segurança no ambiente hospitalar, de modo a preservar a integridade dos pacientes e a qualidade da assistência prestada.

Frente ao exposto, esta pesquisa busca responder: Quais são as características das quedas de pacientes em hospital especializado em cardiologia?

Para tanto, objetiva-se caracterizar as quedas de pacientes, segundo registro de eventos adversos, em unidades de internação de hospital especializado em cardiologia, do município de São Paulo. 


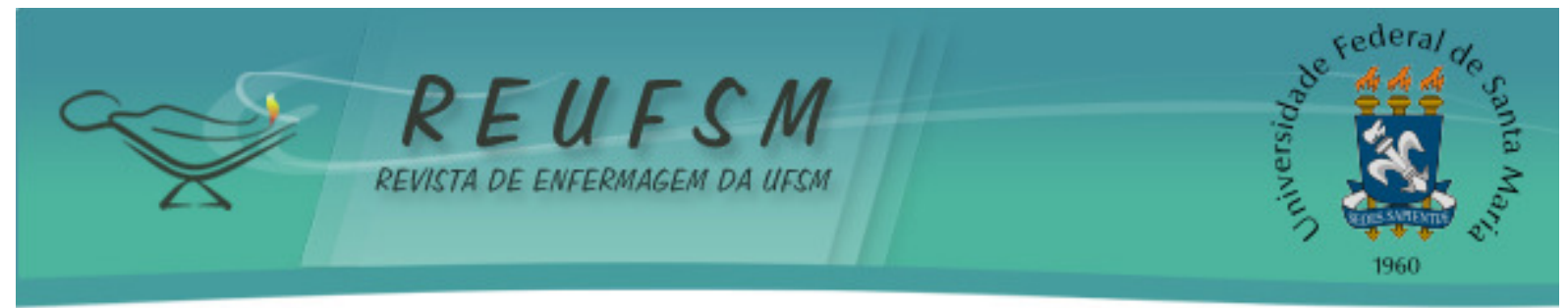

MÉTODO

Trata-se de estudo retrospectivo, descritivo e exploratório realizado em hospital público, especializado em cardiologia do município de São Paulo (SP). A referida instituição é credenciada para atendimento de pacientes que requerem procedimentos de alta complexidade, provenientes do Sistema Único de Saúde (SUS), beneficiários de convênios, seguros médicos e particulares. Além disso, destaca-se também pelas atividades de ensino e pesquisa.

A coleta dos dados foi realizada em 2009, com registros referentes aos meses de janeiro a junho de 2007, computando um total de 67 quedas. A coleta de dados, neste período, ocorreu em virtude de um estudo institucional realizado pela pesquisadora. Para tanto, foram utilizados os formulários de notificação de eventos adversos, padronizados pela coordenação de enfermagem e preenchidos pelos enfermeiros das unidades de internação no momento da ocorrência da queda. Informações complementares ao estudo, como dias de internação e medicações em uso foram obtidas por meio de consulta ao prontuário.

Os dados foram armazenados em banco de dados no Microsoft Excel, processados no programa Statistical Package for the Social Sciencies (SPSS) 15.0 for Windows, analisados descritivamente e apresentados em frequências absolutas e relativas. 0 controle de qualidade dos dados da planilha foi efetuado por um dos autores desta pesquisa.

Para cálculo da incidência de queda utilizou-se a fórmula ${ }^{13}$ : Incidência de queda de paciente $=n^{\circ}$ de quedas $/ n^{\circ}$ paciente-dia $\times 1000$. Cabe esclarecer que também adotou-se, neste estudo, a definição de queda preconizada pelo Núcleo de Apoio a Gestão Hospitalar (NAGEH). ${ }^{13}$

O estudo foi aprovado pelo Comitê de Ética em Pesquisa da instituição, sob protocolo $\mathrm{n}^{\circ}$ 0683/06 e o desenvolvimento atendeu as normas nacionais e internacionais de ética em pesquisa envolvendo seres humanos.

\section{RESULTADOS}

Durante o período do estudo, foram registrados 67 eventos relacionados à queda nas unidades de internação, média de 11,1 ao mês. Considerando-se o total de 32,833 pacientes-dia, a incidência deste evento foi de 2,04/1,000 pacientes.

Tabela 1 - Ocorrências de quedas de pacientes, segundo sexo, faixa etária e dias de internação. São Paulo, SP, 2007.

\begin{tabular}{llc}
\hline Características & $\mathrm{n}$ & $\%$ \\
\hline Sexo & & 59,7 \\
Masculino & 40 & 40,3 \\
Feminino & 27 & 6,0 \\
Faixa etária (anos) & & 4,4 \\
< 30 & 04 & 6,0 \\
30 a 39 & 03 & 19,4 \\
40 a 49 & 04 & 22,4 \\
50 a 59 & 13 & 41,8 \\
60 a 69 & 15 & 58,2 \\
$70+$ & 28 & 32,8 \\
Dias de internação & & 9,0 \\
0 a 30 & 39 & \\
31 a 60 & 22 & \\
61 a 90 & 06 & \\
\hline
\end{tabular}

Maior frequência de queda foi verificada com pacientes do sexo masculino $(59,7 \%)$, predominantemente na faixa etária de 60 anos ou mais, com média de idade de 


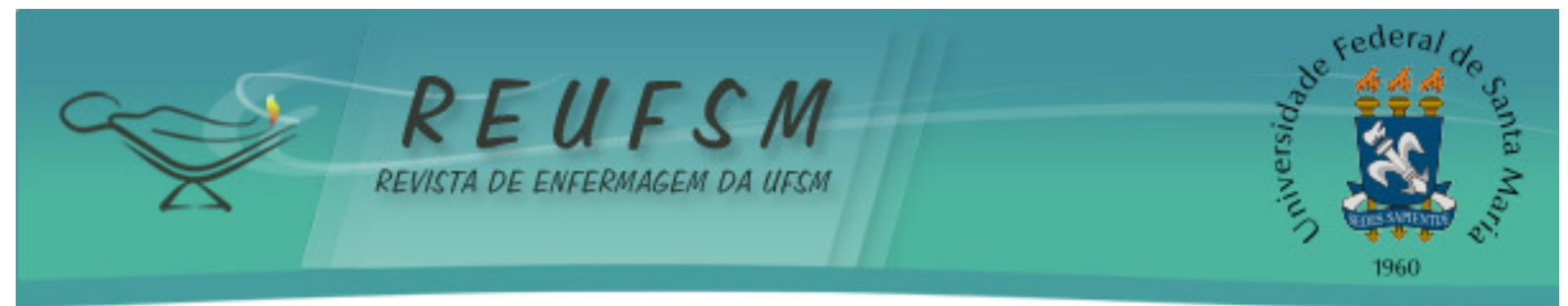

64,04 anos. Estas, ocorridas, na sua maioria, nos primeiros 30 dias da internação $(58,2 \%)$, conforme Tabela 1.

No que se refere às medicações, $60(89,5 \%)$ pacientes faziam uso de antihipertensivos, 51 (76,1\%) psicotrópicos, $46(68,6 \%)$ antiagregante plaquetário, oito $(11,9 \%)$ digitálicos, e cinco $(7,4 \%)$ usavam nitratos e neuroplépticos $(7,4 \%)$.

As quedas da própria altura foram as mais frequentes, com 43 (64,2\%) episódios, seguidas pelas de poltrona $(18,0 \%)$ e do leito $(10,4 \%)$. Verifica-se que os eventos predominaram no período noturno, com $37(55,2 \%)$ quedas. Quanto ao local de ocorrência, o quarto obteve maior destaque $(73,1 \%)$, em segundo lugar o banheiro $(22,4 \%)$, Tabela 2.

\begin{tabular}{lcc}
\hline Tipo de queda & $\mathbf{n}$ & $\%$ \\
Leito & 07 & 10,4 \\
Poltrona & 12 & 18,0 \\
Própria altura & 43 & 64,2 \\
Acidental (ambiente) & 05 & 7,4 \\
Horário & & \\
Manhã & 19 & 28,4 \\
Tarde & 11 & 16,4 \\
Noite & 37 & 55,2 \\
Local & & \\
Quarto & 49 & 73,1 \\
Banheiro & 15 & 22,4 \\
Corredor & 03 & 4,5 \\
\hline
\end{tabular}

Do total de ocorrências (67), 27 pacientes tinham a presença de um acompanhante no momento das quedas. Segundo registro, tinha como recomendação da equipe de enfermagem repouso absoluto, três $(4,5 \%)$ pacientes, levantar com auxílio, 48 $(71,6 \%)$ e liberado para levantar sozinho, $16(23,9 \%)$.

\section{DISCUSSÃO}

No âmbito das atribuições da enfermagem a prevenção de danos deve ser elencada como prioridade, pois pode resultar em prejuízos para o paciente, instituição e equipe. Portanto, cabe ao enfermeiro estabelecer relação terapêutica, pautada em ações educativas e propedêuticas, aplicando a sistematização da assistência de enfermagem.

Neste contexto, vale salientar que o ambiente hospitalar é hostil, impessoal e não isento de iatrogenias. As quedas representam $70 \%$ das ocorrências no ambiente hospitalar ${ }^{4}$ e evidencia-se também que $2 \%$ dos pacientes sofrem algum tipo de queda durante a hospitalização. ${ }^{14}$

No presente estudo o sexo masculino foi predominante $(59,7 \%)$, divergente dos dados de outra pesquisa em que o feminino foi apontado em $68,2 \%$ dos casos de queda ${ }^{15}$, e considerado como variável importante pela sua prevalência. ${ }^{16}$

No entanto, a predominância do sexo masculino encontrada neste estudo pode ser devida às características da instituição em que foi realizada a pesquisa. No Brasil as doenças cardiovasculares têm prevalência maior entre homens, em virtude de não priorizarem os cuidados com a própria saúde tanto quanto as mulheres. ${ }^{17}$ Além disso, os mesmos solicitam menos auxílio para realização de atividades da vida diária. ${ }^{18}$

Outro fator a considerar é a idade avançada, pois aumenta a vulnerabilidade e a susceptibilidade a doenças, ${ }^{8}$ corroborando os achados desta pesquisa em que $28(41,8 \%)$ dos pacientes tinham 70 anos ou mais. Resultados semelhantes foram encontrados em estudo $^{6}$ em que a ocorrência de queda predominou em homens, na faixa etária entre $76 \mathrm{a}$ 


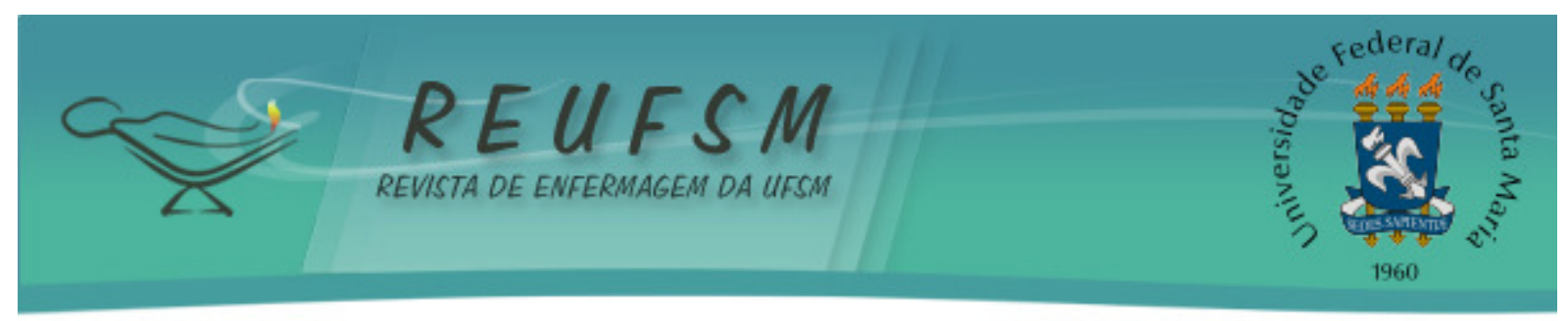

80 anos, e que tinham doenças cardiovasculares (47,3\%). A idade contribui para queda, visto que o envelhecimento causa déficit motor, alterações visuais, posturais, auditivas, vestibulares e dos reflexos. ${ }^{10}$ No presente estudo verificou-se que, $43(64,2 \%)$ dos pacientes tinham idade igual ou superior a 60 anos e sofreram queda da própria altura.

Outro dado evidenciado nesta pesquisa refere-se ao predomínio de quedas nos primeiros 30 dias de internação (58,2\%). Dados estes corroborados em estudo que analisou 826 quedas ocorridas em hospital terciário e, destas, 61,7\% incidiram nos primeiros cinco dias de hospitalização. ${ }^{5}$ Este fato pode ser atribuído ao período de adaptação ao ambiente hospitalar, às cirurgias, reações adversas de medicações e/ou pela dificuldade de aceitar sua dependência à equipe de enfermagem.

Em relação às medicações utilizadas pelos pacientes incluídos nesta pesquisa, a maioria contribui para aumentar o risco de quedas em decorrência dos eventos adversos hemodinâmicos e sensoriais, embora a queda no ambiente hospitalar seja considerada multifatorial. ${ }^{19-20}$

Neste contexto, os medicamentos com repercussões fisiológicas merecem atenção da equipe de enfermagem no sentido de prevenir a ocorrência de quedas. Para que o profissional consiga ter esse olhar deve possuir conhecimentos e aplicar a sistematização de enfermagem, ferramenta indispensável para implementação de assistência individualizada e pautada nas demandas de cuidado. ${ }^{21}$

Além disso, a participação das instituições hospitalares no processo de certificação em saúde estimula discussões sobre as questões de segurança e de desenvolvimento de estratégias que permitam aos profissionais identificar e reduzir os riscos a que os pacientes estão expostos durante a internação, consequentemente refletindo numa assistência de maior qualidade. ${ }^{7}$ Dado corroborado em pesquisa que avaliou a implantação de protocolo de gerenciamento de quedas em um hospital privado. Neste estudo, o processo de acreditação direcionou a efetivação das ações preventivas e norteou o acompanhamento do indicador de qualidade assistencial de incidência de queda na instituição. ${ }^{22}$

Durante a hospitalização prioriza-se atender às necessidades de segurança e conforto, além de outras prejudicadas com a internação. Ações estas que conferem credibilidade à equipe, despertando no paciente sentimentos de confiança, estabilidade, dependência e proteção, assim como o bem-estar. No entanto, ressalta-se que para atender às necessidades de forma integralizada é necessário empenho dos gestores das instituições de saúde para atender satisfatoriamente a demanda da clientela.

Assim, o gerenciamento deve estar pautado em indicadores de qualidade assistencial que propiciarão avaliação dessa atividade, permitindo análise comparativa entre os fatos reais e as metas a serem alcançadas, conferindo dados que permitirão reconhecer parâmetros inter-relacionados em uma organização. Este processo merece olhar direcionado ao problema em questão com a possibilidade de analisar, revisar, propor soluções e avaliar situações que necessitam de intervenções educativas e preventivas.

Nesse sentido, a queda de pacientes hospitalizados configura um problema sério e comum nas instituições de saúde, que não pode ser analisado como fato isolado e superficial. Portanto, quando o indivíduo encontra-se hospitalizado deve-se considerar os fatores intrínsecos, como alterações fisiológicas que podem surgir em consequência do processo de envelhecimento, alterações patológicas e efeitos colaterais da terapêutica medicamentosa, dentre outras. ${ }^{23}$ Além desses, destacam-se os fatores extrínsecos, que podem gerar atitudes e comportamentos peculiares e não costumeiros causando quedas. ${ }^{24}$

Considerando esses fatores extrínsecos, deve ser priorizada a atenção principalmente aos idosos que apresentam deficiência no equilíbrio, na marcha e quando estes se propõem a realizar atividades que envolvem riscos de queda. Neste contexto, o 


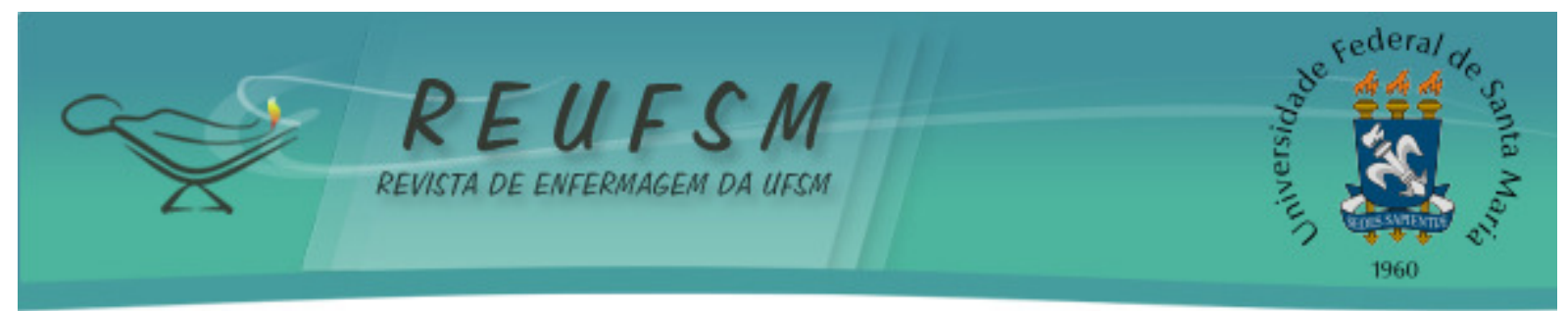

ato de escorregar, tropeçar, pisar em falso, trombar em objetos constituem situações que propiciam a queda da pessoa idosa.

As grades de proteção são componentes de segurança que todos os leitos deveriam conter, porém na realidade não é isso que ocorre na maioria dos hospitais brasileiros. Por esse fato, a evolução do estado do paciente deve ser diária e a observação contínua durante todo o período de internação, principalmente para aqueles pacientes que apresentam fatores de risco de queda. Além disso, é de fundamental importância a informação e orientação do paciente e dos acompanhantes para prevenção de queda.

A cadeira de rodas é um dos meios de transporte de pacientes no âmbito hospitalar. Na literatura não foram encontrados relatos de quedas durante o seu uso, embora seja passível de ocorrência. Neste estudo não houve notificação de quedas relacionadas ao uso da cadeira de rodas e sim ao da poltrona hospitalar utilizada nos quartos da referida instituição $(18,0 \%)$. Essa poltrona permite que o paciente fique em posição semi-deitado confortavelmente com as pernas elevadas, é segura quando permanece nesta posição. Porém, caso se levante sem auxílio é possível queda da mesma, devido à perda de sustentação e estabilidade.

$\mathrm{Na}$ percepção do paciente, a micção é encarada como urgência devido ao desconforto que pode surgir. Nesse impulso, ele tenta realizar suas necessidades sem solicitar apoio da equipe de enfermagem ocultando a sua dependência. É fato que a enfermagem é constituída predominantemente por profissionais do sexo feminino, e muitos pacientes do sexo masculino, diante disto, no sentido de preservar sua intimidade, não solicitam ajuda, contribuindo para episódios de queda da poltrona. Em nosso estudo 48 $(71,6 \%)$ dos pacientes que sofreram queda tinham recomendação de levantar com auxílio, porém isto não ocorreu.

Outros fatores relacionados ao ambiente propiciam a queda, tanto no domicílio quanto no hospital, em diferentes locais. Geralmente o revestimento é de material derrapante, escorregadios, desprovidos de barras de apoio, com iluminação deficiente, o que facilita o desequilíbrio provocando a queda.

Nesta casuísta, 35,8\% dos pacientes se encontravam acompanhados, e, mesmo assim, sofreram quedas. Os dados deste estudo mostraram que a presença do acompanhante para idosos, embora assegurada pela legislação, ${ }^{25}$ não impede a ocorrência deste evento adverso. Visto que, dos 37 pacientes que caíram no período noturno, $50 \%$ estavam acompanhados. Nesta pesquisa, não foi possível verificar se no momento da ocorrência o acompanhante se fazia presente para auxiliar o paciente a levantar ou a deambular.

\section{CONCLUSÕES}

Constatou-se que as quedas predominaram em idosos, com idade igual ou superior a 60 anos, sexo masculino, e que no âmbito da cardiologia também são multifatoriais.

Os resultados este estudo também denotam a necessidade de se ter mais cuidado com os idosos internados, durante o período noturno, no quarto e especialmente nos primeiros trinta dias de internação. As contribuições fornecidas pelos registros de eventos adversos facilitaram a identificação dos fatores de risco para quedas demonstrando a necessidade de se propor intervenções preventivas, uma vez que assumir o evento e identificar as causas são maneiras de praticar a segurança do paciente.

Mediante à relevância do tema, propõe-se o desenvolvimento de futuros estudos longitudinais e multicêntricos, com vistas a aumentar o poder de generalização do mesmo. 


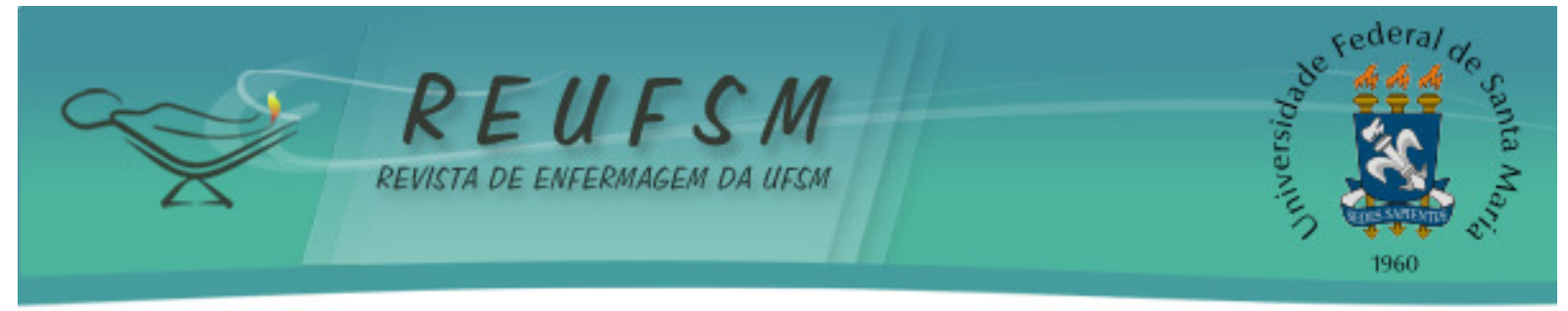

\section{REFERÊNCIAS}

1. Brasil. Ministério da Saúde. Secretaria de Atenção à Saúde. Departamento de Ações Programáticas e Estratégicas. Atenção à saúde da pessoa idosa e envelhecimento. Brasília (DF); 2010.

2. Santos SSC. Concepções teórico-filosóficas sobre envelhecimento, velhice, idoso e enfermagem gerontogeriátrica. Rev Bras Enferm. 2010;63(6):1035-9.

3. Ferreira OGL, Maciel SC, Silva AO, Santos WS, Moreira MASP. $O$ envelhecimento ativo sob o olhar de idosos funcionalmente independentes. Rev Esc Enferm USP. 2010;44(4):1065-9.

4. Carneiro FS, Bezerra ALQ, Silva AEBC, Souza LP, Paranaguá TTB, Branquinho NCSS. Eventos adversos na clínica cirúrgica de um hospital universitário: instrumento de avaliação da qualidade. Rev Enferm UERJ. 2011;19(2):204-11.

5. Paiva MCMS, Paiva SAR, Berti HW, Campana AO. Caracterização das quedas de pacientes segundo notificação em boletins de eventos adversos. Rev Esc Enferm USP. 2010;44(1):134-8.

6. Filgueiras MC, Santiago FR, Santiago HAR, Vieira LJES. Fraturas em idosos decorrentes de quedas registradas em hospital terciário de referência em traumatologia no ano de 2004. Rev Bras Promoç Saúde. 2007;20(4):226-32.

7. Luzia MF, Victor MAG, Lucena AF. Diagnóstico de enfermagem risco de quedas: prevalência e perfil clínico de pacientes hospitalizados. Rev Latinoam Enferm. 2014;22(2):262-8.

8. São Paulo (Estado). Secretaria de Estado da Saúde. Vigilância e prevenção de quedas em idosos. Louvison MCP, Rosa TEC, editores. São Paulo: SES/SP; 2010.

9. Tinetti ME. Clinical practice: preventing falls in elderly persons. $N$ Engl Med. 2003;348(1):42-9.

10. Pinho TAM, Silva AO, Tura LFR, Moreira MASP, Gurgel SN, Smith AAF, et al. Avaliação do risco de quedas em idosos atendidos em Unidade Básica de Saúde. Rev Esc Enferm USP. 2012;46(2):320-7.

11. Abreu C, Mendes A, Monteiro J, Santos FR. Quedas em meio hospilalar: um estudo longitudinal. Rev Latinoam Enferm. 2012;20(3):597-603.

12. Moura GMSS, Juchem BC, Falk MLR, Magalhães AMM, Suzuki LM. Construção e implantação de dois indicadores de qualidade assistencial de enfermagem. Rev Gaúch Enferm. 2009;30(1):136-40.

13. Compromisso com a Qualidade Hospitalar (CQH). Manual de indicadores de enfermagem NAGEH [Internet]. $2^{a}$ ed. São Paulo (SP): APM/CREMESP; 2012 [acesso em 2013 jan 26]. Disponível em: http://www.cqh.org.br/portal/pag/doc.php?p_ndoc=125.

14. Hartikainen S, Lonnroos E, Louhivuori K. Medication as a risk for falls: critical systematic review. J Gerontol A Biol Med Sci. 2007;62(10):1172-81.

15. Paula FL, Fonseca MJM, Oliveira RVC, Rozanfeld S. Perfil de idosos com internação por quedas nos hospitais públicos de Niterói (RJ). Rev Bras Epidemiol. 2010;13(4):587-95.

16. Moreira MD, Costa AR, Felipe RL, Caldas CP. Variáveis associadas à ocorrência de quedas a partir dos diagnósticos de enfermagem em idosos atendidos ambulatorialmente. Rev Latinoam Enferm. 2007;15(2):311-7. 


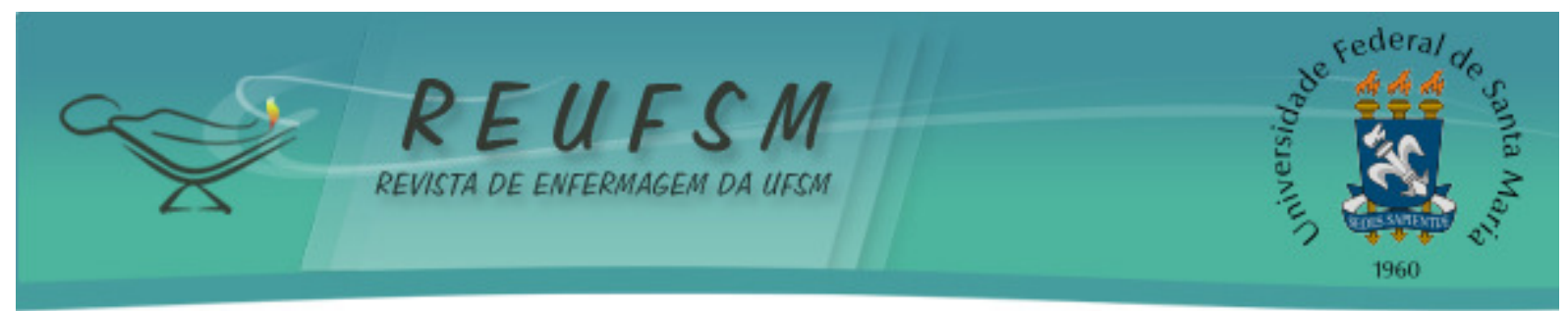

17. Chagas ACP, Zilli EC, Ferreira JFM, Moretti MA, Ramos RF. Saúde cardiovascular do homem brasileiro - visão da Sociedade Brasileira de Cardiologia. Arq Bras Cardiol. 2009;93(6):584-87.

18. Costa SGRF, Monteiro DR, Hemesath MP, Almeida MA. Caracterização das quedas do leito sofridas por pacientes internados em um hospital universitário. Rev Gaúcha Enferm. 2011; 32(4):676-81.

19. Shuto H, Imakyure O, Matsumoto J, Egawa T, Jiang $Y$, Hirakawa M, et al. Medication use as risk factor for inpatient falls in a acute care hospital: a case-crossover study. $\mathrm{Br} \mathrm{J}$ Clin Pharmacol. 2010;69(5):535-42.

20. Schwendimann R, Bühler H, De Geest S, Milisen K. Caracteristics of hospital in patient falls across clinical departments. Gerontology. 2008;54(6):342-8.

21. Silva LG, Jodas DA, Baggio SC, Vituri DW, Matsuda LM. Prescrição de enfermagem e qualidade do cuidado: um estudo documental. Rev Enferm UFSM [Internet]. 2012 jan-abr [acesso em 2014 maio 5];2(1):97-107. Disponível em: http://cascavel.ufsm.br/revistas/ojs2.2.2/index.php/reufsm/article/view/4546/3131.

22. Correa AD, Marques IAB, Martinez MC, Laurino OS, Leão ER, Chimentão DMN. Implantação de um protocolo para gerenciamento de quedas em hospital: resultados de quatro anos de seguimento. Rev Esc Enferm USP. 2012;46(1):67-74.

23. Menezes RL, Bachion MM. Estudo da presença de fatores de risco intrínsecos para quedas em idosos institucionalizados. Cienc Saúde Coletiva. 2008;13(4):1209-18.

24. Freitas R, Santos SSC, Hammerschmidt KSA, Silva ME, Pelzer MT. Cuidado de enfermagem para prevenção de quedas em idosos: proposta para ação. Rev Bras Enferm. 2011;64(3):478-85.

25. Brasil. Presidência da República. Lei $n^{\circ} 10.741$, de $1^{\circ}$ de outubro de 2003. Dispõe sobre o Estatuto do Idoso e dá outras providências. Brasília (DF); 2003.

Data de recebimento: $16 / 04 / 2014$

Data de aceite: $27 / 11 / 2014$

Contato com autor responsável: Silmara Meneguin

Endereço postal: Faculdade de Medicina de Botucatu - Departamento de Enfermagem Distrito de Rubião Jr. s/n - CEP 18618-970

E-mail: silmeneguin@fmb.unesp.br 